\title{
Acute Pulmonary Embolism, Multiple Coronary Thrombosis, and Thrombi in the Left Ventricle and Ascending Aorta in a Patient with COVID-19 Infection
}

\author{
David Chipayo Gonzales' ${ }^{1}$ Paloma Pérez Espejo², Breda Hennessey¹, Javier Fernández Portales², \\ Sebastián Romani ${ }^{2}$, Iván Núñez Gil ${ }^{1}$ \\ ${ }^{1}$ Hospital Clínico San Carlos, Madrid, Spain \\ ${ }^{2}$ Complejo Universitario de Cáceres, Caceres, Spain \\ Email: davidchipayogz@outlook.es,paloma.perez@salud-juntaex.es,Breda_82@hotmail.com,portales70@hotmail.com, \\ sebastian.romani@hotmail.com, ibnsky@yahoo.es
}

How to cite this paper: Gonzales, D.C., Espejo, P.P., Hennessey, B., Portales, J.F., Romani, S. and Gil, I.N. (2021) Acute Pulmonary Embolism, Multiple Coronary Thrombosis, and Thrombi in the Left Ventricle and Ascending Aorta in a Patient with COVID-19 Infection. Case Reports in Clinical Medicine, 10, 314-322.

https://doi.org/10.4236/crcm.2021.1010040

Received: September 8, 2021

Accepted: October 18, 2021

Published: October 21, 2021

Copyright (๑) 2021 by author(s) and Scientific Research Publishing Inc. This work is licensed under the Creative Commons Attribution International License (CC BY 4.0).

http://creativecommons.org/licenses/by/4.0/

\begin{abstract}
Both arterial and venous thromboembolic events are common in patients hospitalized in intensive care units with severe COVID-19. These patients often have laboratory findings consistent with a hypercoagulable state, suggesting widespread thrombosis and fibrinolysis, as well as elevated levels of D-dimer, von Willebrand factor (VWF), and factor VIII. There is increasing evidence that these thromboembolic events are associated with worse outcomes. We present the case of a 61-year-old man admitted for bilateral pneumonia due to COVID-19 infection, who developed during his hospitalization; Bilateral pulmonary thromboembolism, an acute myocardial infarction due to multiple coronary thrombosis, an intracavitary thrombus and thrombus in the ascending aorta. The patient was treated with systemic fibrinolysis and full doses of anticoagulation for pulmonary embolism, an emergency primary percutaneous coronary intervention (PCI) was performed with the implant of a drug eluting stent (DES) in the left anterior descending artery (LAD). Triple therapy with aspirin, clopidogrel and heparin was maintained until discharge. After the patient discharge, aspirin was stopped and treatment with clopidogrel and dabigatran was maintained for three months. In a subsequent outpatient control, the thrombi of the left ventricle and the ascending aorta resolved.
\end{abstract}

\section{Keywords}

COVID 19 Infection, Pulmonary Embolism (PE), ST-Segment Elevation 
Myocardial Infarction (STEMI), Intracoronary Thrombosis, Intra-Aortic Thrombus

\section{Introduction}

The SARS COV 2 virus infection is responsible for more than 4 million deaths worldwide since the outbreak of late 2019 [1]. The range of clinical responses to severe acute respiratory syndrome coronavirus 2 (SARS-CoV-2) infection is extremely broad. Although most patients with coronavirus disease 2019 (COVID-19) present with a mild upper respiratory tract infection and then recover, some infected patients develop pneumonia, acute respiratory distress syndrome, multi-organ failure, and death. Clues to the pathogenesis of severe COVID-19 lie in the systemic inflammation and thrombosis observed in infected patients [2]. The first cohorts of patients with covid 19 described predominantly pulmonary involvement with the development of bilateral pneumonia, especially in patients older than 75 years or with cardiovascular risk factors. According to some studies, the overall mortality of patients with COVID-19 infection is between $4.2 \%$ and $13.4 \%$. And in patients admitted to the ICU, mortality can rise to $34.8 \%$ [3]. This high mortality was initially explained by the development of ARDS (similarto what has been described in other respiratory viruses) and the impact of concurrent infections in critically ill COVID-19 patients (Influenza, HIV, hepatitis virus, Klebsiella, pneumococcus, and others) [4]. However, currently, increasing evidence exists of the close relationship between severe COVID-19 infection and the appearance of venous thromboembolisms, myocardial infarction strokes and others vascular phenomena [5]. Both arterial and venous thromboembolism are common in patients with severe COVID-19. The incidence of venous thromboembolic events in patients with COVID-19 admitted to intensive care units ranges from $20 \%$ to $35 \%$, and deep venous thrombosis has been identified in $70 \%$ to $100 \%$ of patients who died from COVD-19 Furthermore, arterial thrombosis resulting in stroke or myocardial infarction occurs in up to $4 \%$ of patients with COVID-19 hospitalized in intensive care units. Patients with severe COVID-19 often have laboratory findings consistent with a hypercoagulable state, suggesting widespread thrombosis and fibrinolysis, as well as elevated levels of D-dimer, von Willebrand factor (VWF), and factor $\mathrm{VIII}^{2}$. These patients also manifest a "cytokine storm" [5], characterized by elevated levels of inflammatory markers such as C-reactive protein and interleukin-6, which have been linked to severity of pneumonia and mortality [6]. The endothelial injury is probably the underlying mechanism that might link inflammation and thrombosis in severe COVID-19 [5] [6]. Autopsy studies have suggested that both endothelial inflammation and microvascular thrombosis are prominent, with inflammatory cells attached to the endothelium of small vessels in lung, kidney, heart, and liver [7]. Microvascular thrombosis with activation, microvascular in- 
flammation, and activation of the complement attack complex (C5b-9) suggest that microvascular injury may be a common trigger for both the inflammatory and thrombotic complications of COVID-19 [6]. There is increasing evidence that anticoagulant treatment reduces mortality in patients with severe COVID-19 infections [7]. Although the strategy (prophylactic vs therapeutic dose) and duration are not well established. Furthermore, even if the thrombotic phenomena are frequent, is rare that all these thromboembolic complications occur in the same patient at the same time as in the case of our patient. We believe that this case is relevant as it highlights the close relationship between COVID-19 infection and the development of venous and arterial thromboembolic complications, its frequency, severity, the poor prognosis that it entails for patients and the current evidence on the treatment of these phenomena.

\section{Case Presentation}

A 61-year-old male with a history of diabetes, hypertension and obesity, who presented to the emergency department of a general hospital in January 2021 with fever, dyspnea, and a capillary oxygen saturation of $88 \%$ on room air. Table 1 shows the laboratory parameters. The patient was diagnosed with bilateral pneumonia do to COVID-19 infection confirmed by PCR test. He was admitted and treated with oxygen, remdesivir and corticoids with a good initial response to treatment. However, 7 days after the admission the patient had a syncopal episode, associated with hypotension and a new onset of sudden dyspnea. A CT thorax scan was performed, and he was diagnosed with a pulmonary embolism with a large thrombus that affected the bifurcation of the main pulmonary artery, and bilateral pulmonary infarction (Figure 1 (a) and Figure 1(b)). Due to

Table 1. Blood tests results at hospital admission.

\begin{tabular}{|c|c|c|}
\hline Parameter & result & normal value \\
\hline Hemoglobin & $14.1 \mathrm{~g} / \mathrm{dl}$ & $13.5-17.5 \mathrm{~g} / \mathrm{dl}$ \\
\hline white blood cells & $5.3 \mathrm{mil} / \mathrm{mm}^{3}$ & $4.5-11 \mathrm{mil} / \mathrm{mm}^{3}$ \\
\hline Platelets & $210 \mathrm{mil} / \mathrm{mm}^{3}$ & $150-450 \mathrm{mil} / \mathrm{mm}^{3}$ \\
\hline Creatinine & $0.89 \mathrm{mg} / \mathrm{dl}$ & $0.7-1.2 \mathrm{mg} / \mathrm{d}$ \\
\hline Troponin $\mathrm{T}$ & $8 \mathrm{ng} / \mathrm{ml}$ & $<14 \mathrm{ng} / \mathrm{ml}$ \\
\hline B-type natriuretic peptide & $96 \mathrm{pg} / \mathrm{ml}$ & $<300 \mathrm{pg} / \mathrm{ml}$ \\
\hline $\mathrm{PCR}^{\star}$ level & 5 & $<10$ \\
\hline $\mathrm{ANA}^{*}$ level & 0.2 & $<1$ \\
\hline ANTI DNA level & neg. & neg \\
\hline C3 complement & 93 & $82-160$ \\
\hline Epstein barr antibodies IG M & neg & neg \\
\hline Parvovirus B19 antibodies IG M & neg & neg \\
\hline HIV antibodies & neg & neg \\
\hline Coxsackie virus antibodies IG M & neg & neg. \\
\hline
\end{tabular}




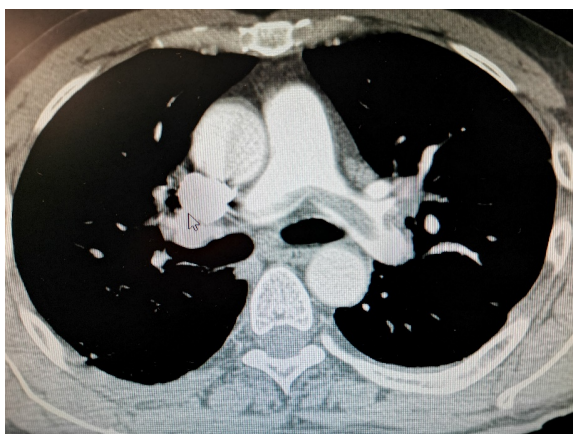

(a)

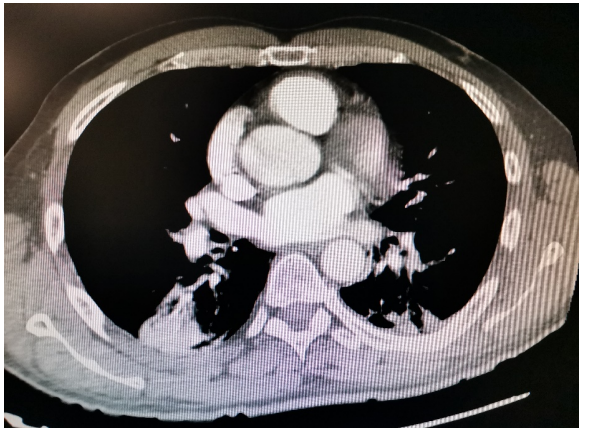

(b)

Figure 1. (a) A CT thorax scan with a large thrombus in the bifurcation of the main pulmonary artery. (b) Bilateral pulmonary infarction with predominant involvement of the inferior lobe of the right lung.

severely dyspnea, desaturation and hypotension, the patient was intubated and ventilated, he received systemic fibrinolytic treatment with alteplase and was anticoagulated with enoxaparin which was started at a dose of $1 \mathrm{mg}$ per kilogram b.i.d. Following 5 days in the ICU with good initial clinical course, the patient was extubated, and transferred to the COVID-19 ward. 20 days after his admission, the patient develop oppressive chest pain and the 12-leads ECG taken after the onset of symptoms showed a ST elevation in I, aVL and the precordial leads from V2-V6 (Figure 2(a)). An emergent cardiac catheterization was performed, and we found ectasic coronary arteries with a thrombotic occlusion in the middle segment of the left anterior descending (LAD) artery, which was the culprit vessel. We also found distal thrombi in the marginal obtuse artery as well as the right coronary artery (Figure 2(b) and Figure 2(c)). We performed thrombus aspiration and a large red thrombus was extracted from the LAD (Figure 2(d)). During the procedure, an intravenous infusion of the glycoprotein IIb-IIIa inhibitor eptifibatide was started and a drug eluting stent (DES) was implanted in the LAD. Immediately following the stent implantation, the patient developed no reflow phenomena, with a poor response to intracoronary adenosine and nitroprusside. The treatment result was a suboptimal with a final TIMI II flow result in the final distal coronary artery, although the patient's chest pain was resolved, with resolution of the ST-segment elevation. A transthoracic echocardiogram performed 2 days after the PCI reported a left ventricular systolic function of 37\% with severe septo-anterior hypokinesis and apical akinesia with a thrombus at the apex of left ventricle (Video 1 and 2 of supplementary materials; links: https://youtu.be/pnBKPuH_KBo and https://youtu.be/sErzRuZQeno) with no intracardiac shunt detected with the echocardiogram bubble test; However, the transthoracic echocardiogram also revealed an image suggestive of a thrombus at the level of the arch of the aorta (Figure 3(a)), which was confirmed by a transesophageal echocardiogram (Figure 3(b) and video 3 of supplementary material; link:

https://youtu.be/BZXKYQ9tTMA) and a further aortic CT scan (Figure 3(c)). 


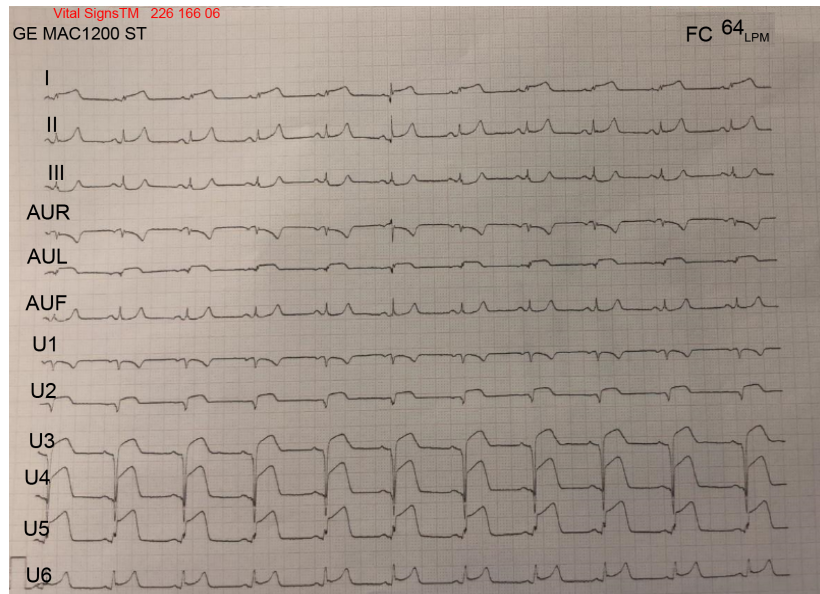

(a)

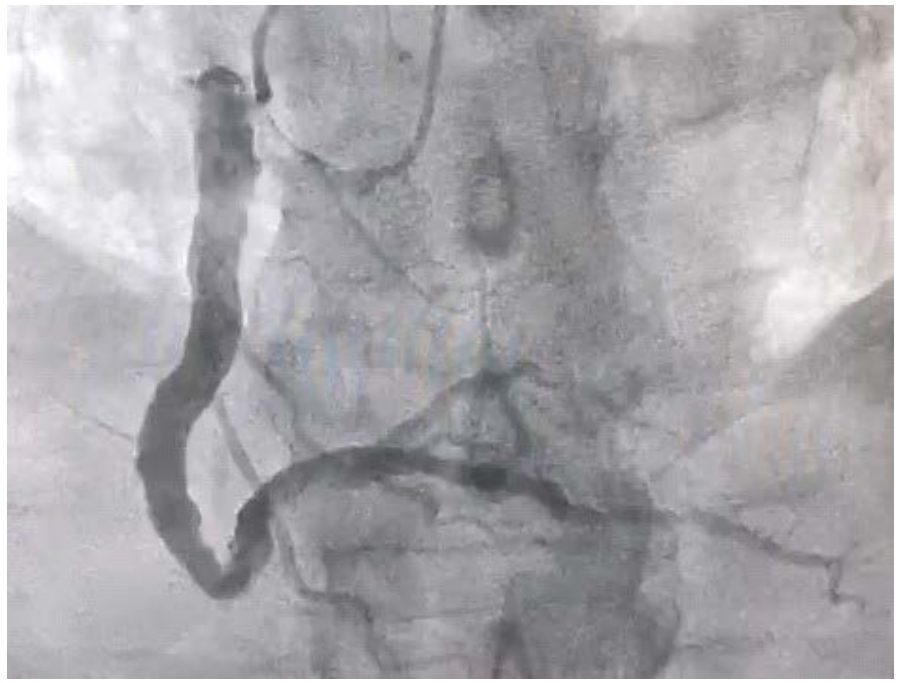

(c)

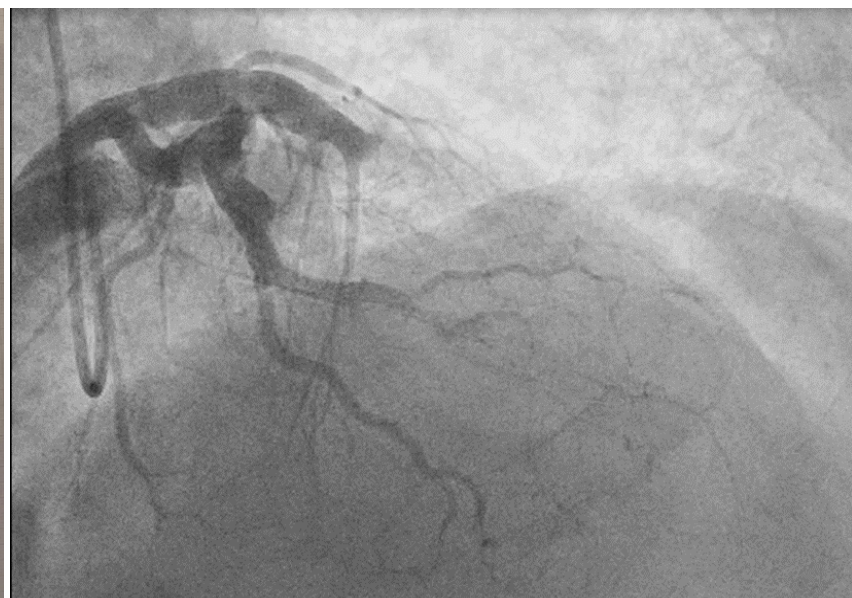

(b)

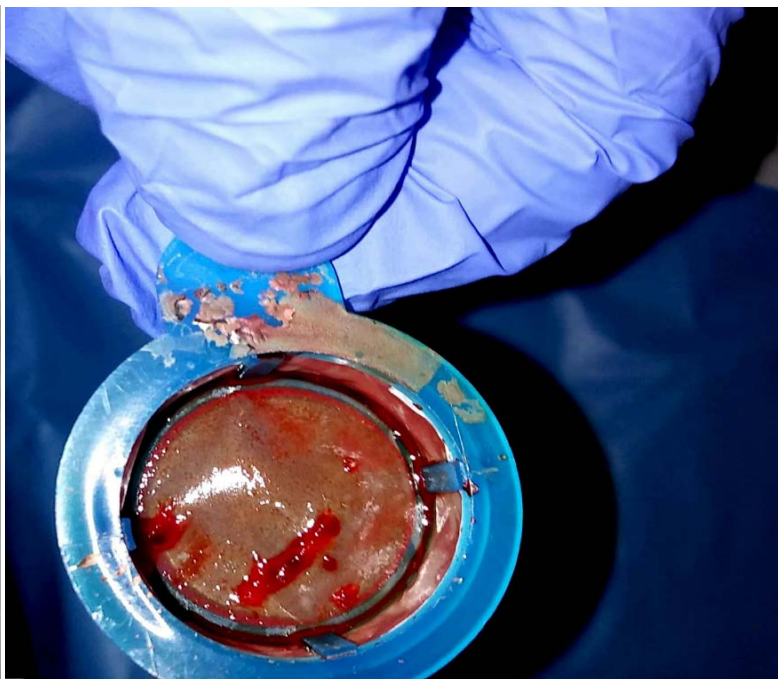

(d)

Figure 2. (a) A 12 leads ecg after the onset of the chest pain shows an ST-segment elevation in I, aVL and precordial leads from V2-V6. (b) Cardiac catheterization with an acute total occlusion of the middle segment of the LAD, and a distal embolus in the Obtuse marginal artery. (c) Tortuous and ectasic right coronary artery with turbulent flow and two focal and severe stenoses in the distal right descending posterior artery. (d) Two red thrombus were aspirated from the middle segment of LAD using the thromboaspiration system. The largest was measured $3.0 \times 19 \mathrm{~mm}$.

No signs of aortic ulcer, wall hematoma, or aortic dissection was found. The previous CT scan was reviewed, and no aortic thrombus was noted. The patient was treated with triple antithrombotic therapy with aspirin $100 \mathrm{mg}$ o.d, clopidogrel $75 \mathrm{mg}$ o.d and enoxaparin $80 \mathrm{mg}$ sc b.i.d until hospital discharge. The type and doses of the antiplatelet agents were chosen following the recommendation of the European guidelines of revascularization in patients who needs triple antithrombotic therapy, and we chose enoxaparin for being the anticoagulant with the most solid evidence at the moment for treatment of the patients with covid 19 and thromboembolic events. along with treatment of myocardial dysfunction with sacubitril valsartan, beta-blockers, mineralocorticoid inhibitors, sodiumglucose cotransporter 2 inhibitors and diuretics on demand. The patient had 


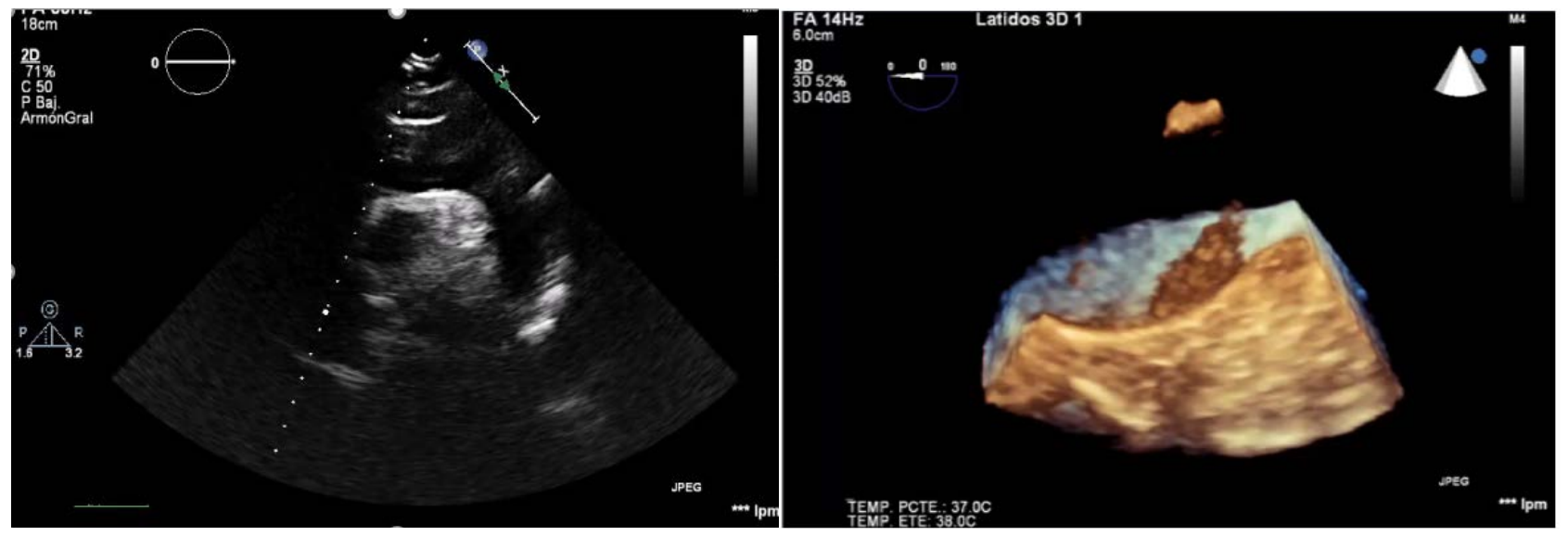

(a)

(b)

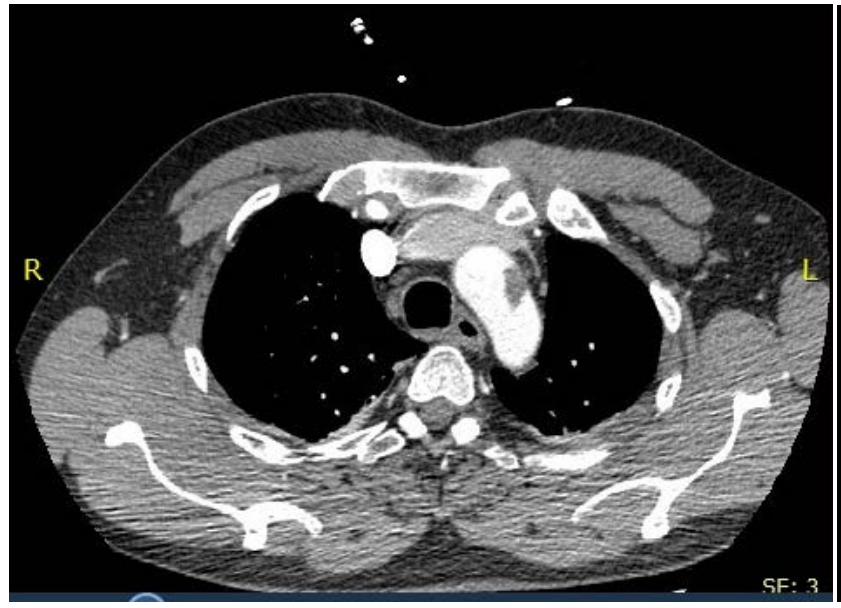

(c)

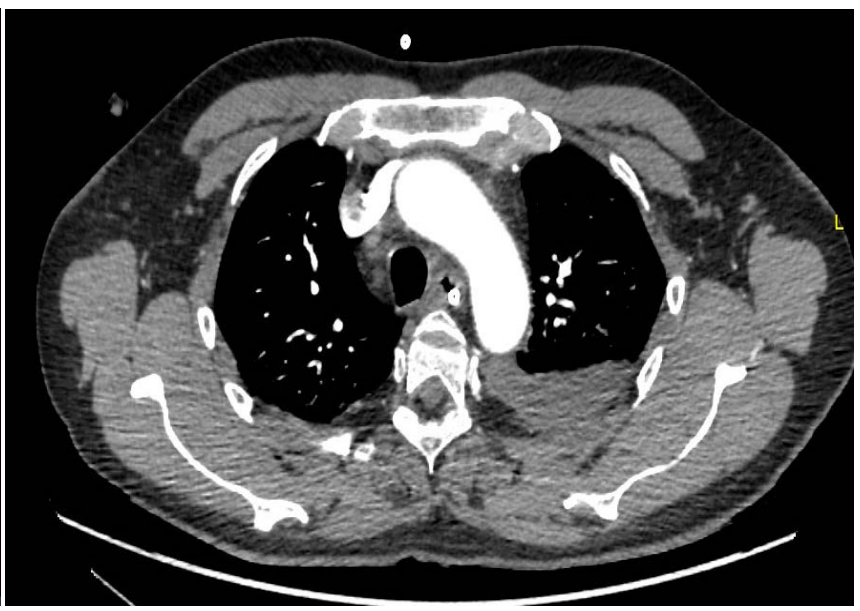

(d)

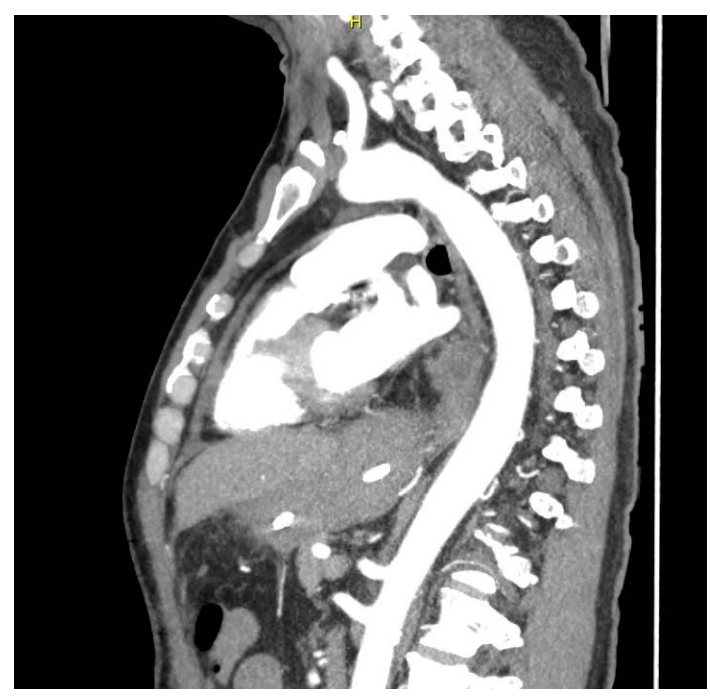

(e)

Figure 3. (a) A TTE image suggestive of thrombus in the aortic arch (blue arrow) on the suprasternal axis plane. (b) Confirmation of the presence of thrombus in the aortic arch in the 3d TEE. (c) An arterial phase of an aorta CT scan with a $7 \mathrm{~mm}$ thrombus adherent to the aortic arch without signs of dissection, intramural hematoma or ulcer of the aortic wall. (d) Control CT scan 3 months after the patient discharge, the axial plane of the aortic arch showing resolution of the thrombus. (e) A sagittal view of the aortic CT scan control at 3 months of discharge showing no residual thrombi or any lesions of the aortic wall. 
a subsequent favorable evolution and was discharged. At the hospital discharge the aspirin was suspended, clopidogrel was maintained and enoxaparin was changed for dabigatran $150 \mathrm{mg}$ b.i.d with outpatient follow up. In the repeat TTE (Video 4 of supplementary material; link: https://youtu.be/07b6F_sZ52M) and CT scan (Figure 3(d)) performed 3 months after the discharge, no thrombus was evident, and patient was asymptomatic.

\section{Discussion}

COVID-19 infection continues to be a major cause of mortality throughout the world. Pulmonary and cardiac injury as indicated by elevated levels of D dimer and cardiac troponins increases risk of death. Although many studies have focused on pulmonary findings of COVID-19, few pathology studies have been conducted specifically examining the effects of COVID-19 on the heart, and most of these did not involve subjects with ST-segment-elevation myocardial infarction or even cardiac injury. The largest published pathology series that included analysis of the lungs, heart and kidneys [8], showed that up to half of the patients present venous pulmonary thromboembolic phenomena and up to $5 \%$ myocardial or coronary microthrombi, and when performing immunofluorecence assay, conglomerates of leukocytes, fibrin, platelets, and the complement attack complex (C5b-9) were sign, associated with high levels of IL6 and IL8 [9]. This inmuno-thrombotic phenomena are more frequently seen in patients with COVID-19 in comparison with other coronaviruses like SARS COV or MERS. And similar with other pathologies as severe sepsis/septic shock and infection by influenza virus H1N1 [10]. The underlying mechanism is likely the endothelial injury that may link inflammation and thrombosis in severe COVID-19 [11]. There are some case reports of thrombotic events in COVID-19 as the. However, to our knowledge, this is one of the few case reports of presentation of multiple venous and arterial thrombotic phenomena in the same patient including multiple intracoronary thrombosis and aortic thrombosis. We raised some hypothesis about the possible explanations for the clinical presentation of our patient, such as the paradoxical embolization of multiple venous thrombi to the left side of the heart, producing coronary, intracardiac and aortic involvement, although it should be mentioned that the echocardiographic bubble test was negative. Another hypothesis was the in-situ formation of intracoronary, intra-aortic and intra-cardiac thrombi secondary to microvascular involvement with severe endothelitis. The third was the formation in-situ of intracoronary thrombi with development of a large antero-apical myocardial infarction, which would generate an apical thrombus that could embolize into the aorta. And finally, a combination of the previously. There is more and more evidence in favor of the anticoagulant treatment to prevent these thromboembolic complications in patients with severe COVID-19 infections [12]. However, it's not clear which strategy (prophylaxis or therapeutic) is optimal in these patients. 


\section{Conclusion}

This case presented a series of thromboembolic complications that can appear in the evolution of patients with severe COVID-19 infections and the way in which they were dealt with. Although we now have a better understanding of the close relationship between COVID-19 infection and cardiovascular involvement and the molecular pathways that are triggered in these patients, little is known about how to prevent and treat them properly. Currently exist poor evidence of the optimal anticoagulant treatment, its dose and duration [13]. As well as which is the optimal antiplatelet therapy for patients with COVID-19 infection who develop STEMI should receive. We believe that further research is needed to determine the correct answers to these questions.

\section{Consent}

The authors confirm that written consent for submission and publication of this case report including image(s) and associated text has been obtained from the patient.

\section{References}

[1] World Health Organization (2021) Coronavirus Disease 2019 (COVID-19): Situation Report, 59. WorldHealth Organization.

https://apps.who.int/iris/bitstream/handle/10665/331475/nCoVsitrep11Mar2020eng .pdf? sequence $=1$ \&isAllowed $=\mathrm{y}$

[2] Klok, F.A., Kruip, M.J.H.A., van der Meer, N.J.M., Arbous, M.S., Gommers, D., Kant, K.M., et al. (2020) Incidence of Thrombotic Complications in Critically Ill ICU Patients with COVID-19. Thrombosis Research, 191, 145-147. https://doi.org/10.1016/j.thromres.2020.04.013

[3] Du, R.H., Liang, L.R., Yang, C.Q., Wang, W., Cao, T.Z., Li, M., Guo, G.Y., Du, J., Zheng, C.L., Zhu, Q., Hu, M., Li, X.Y., Peng, P. and Shi, H.Z. (2020) Predictors of Mortality for Patients with COVID-19 Pneumonia Caused by SARSCoV-2: A Prospective Cohort Study. European Respiratory Journal, 55, Article ID: 2000524. https://doi.org/10.1183/13993003.00524-2020

[4] Sarkar, S., Khanna, P. and Singh, A.K. (2021) Impact of COVID 19 in Patient with Concurrent Coinfection: A Sistemyc Review and a Meta-Analisys. Journal of Medical Virology, 93, 2385-2395. https://doi.org/10.1002/jmv.26740

[5] Varga, Z., Flammer, A.J., Steiger, P., Haberecker, M., Andermatt, R., Zinkernagel, A.S., et al. (2020) Endothelial Cell Infection and Endotheliitis in COVID-19. The Lancet, 395, 1417-1418. https://doi.org/10.1016/S0140-6736(20)30937-5

[6] Driggin, E., Madhavan, M.V., Bikdeli, B., Chuich, T., Laracy, J., Bondi-Zoccai, G., et al. (2020) Cardiovascular Considerations for Patients, Health Care Workers, and Health Systems during the Coronavirus Disease 2019 (COVID-19) Pandemic. Journal of the American College of Cardiology, 75, 2352-2371. https://doi.org/10.1016/j.jacc.2020.03.031

[7] Helms, J., Tacquard, C., Severac, F., Leonard-Lorant, I., Ohana, M., Delabranche, X., et al. (2020) Crics Triggersep Group (Clinical Research in Intensive Care and Sepsis Trial Group for Global Evaluation and Research in Sepsis). High Risk of Thrombosis in Patients in Severe SARS-CoV-2 Infection: A Multicenter Prospective 
Cohort Study. Intensive Care Medicine, 46, 1089-1098.

https://doi.org/10.1007/s00134-020-06062-x

[8] Fox, S.E., Akmatbekov, A., Harbert, J.L., Li, G., Quincy Brown, J. and Vander Heide, R.S. (2020) Pulmonary and Cardiac Pathology in African American Patients with COVID-19: An Autopsy Series from New Orleans. The Lancet Respiratory Medicine, 8, 681-686. https://doi.org/10.1016/S2213-2600(20)30243-5

[9] Lala, A., Johnson, K.W., Januzzi, J.L., Russak, A.J., Paranjpe, I., Richter, F., Zhao, S., Somani, S., Van Vleck, T., Vaid, A., et al. (2020) Mount Sinai COVID Informatics Center. Prevalence and Impact of Myocardial Injury in Patients Hospitalized with COVID-19 Infection. Journal of the American College of Cardiology, 76, 533-546. https://doi.org/10.1016/j.jacc.2020.06.007

[10] Giannisa, D., Ziogasb, L.A. and Giannid, P. (2020) Coagulation Disorders in Coronavirus Infected Patients: COVID-19, SARSCoV-1, MERS-CoV and Lessons from the Past. Journal of Clinical Virology, 127, 104362.

https://doi.org/10.1016/j.jcv.2020.104362

[11] Schaller, T., Hirschbuhl, K., Burkhardt, K., Braun, G., Trepel, M., Markl, B. and Claus, R. (2020) Postmortem Examination of Patients with COVID-19. JAMA, 323, 2518-2520. https://doi.org/10.1001/jama.2020.8907

[12] Paranjpe, I., Fuster, V., Lala, A., et al. (2020) Association of Treatment Dose Anticoagulation with In-Hospital Survival among Hospitalized Patients with COVID-19. Journal of the American College of Cardiology, 76, 122-124. https://doi.org/10.1016/j.jacc.2020.05.001

[13] Zill-e-Huma, R. and Wilkinson, A. (2021) Treatment Strategies for COVID-19 in Pregnancy: Short Review of Current Recommendations. Open Journal of Obstetrics and Gynecology, 11, 823-829. https://doi.org/10.4236/ojog.2021.117076 